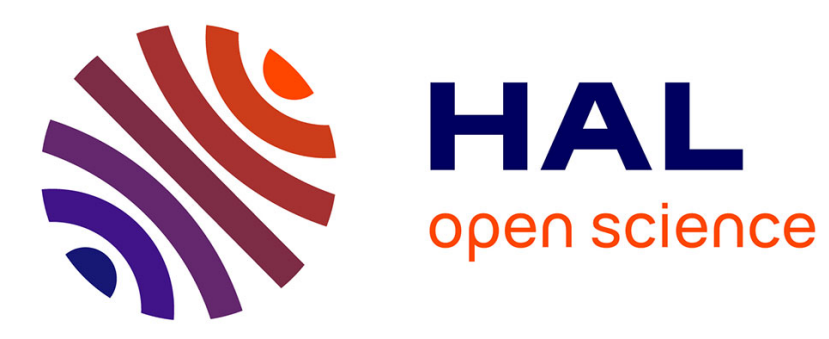

\title{
Interval Estimation for LPV Systems Applying High Order Sliding Mode Techniques
}

Denis Efimov, Leonid Fridman, Tarek Raissi, Ali Zolghadri, Ramatou Seydou

\section{To cite this version:}

Denis Efimov, Leonid Fridman, Tarek Raissi, Ali Zolghadri, Ramatou Seydou. Interval Estimation for LPV Systems Applying High Order Sliding Mode Techniques. Automatica, 2012, 48 (9), pp.23652371. 10.1016/j.automatica.2012.06.073 . hal-00701643

\section{HAL Id: hal-00701643 \\ https://inria.hal.science/hal-00701643}

Submitted on 6 Jun 2012

HAL is a multi-disciplinary open access archive for the deposit and dissemination of scientific research documents, whether they are published or not. The documents may come from teaching and research institutions in France or abroad, or from public or private research centers.
L'archive ouverte pluridisciplinaire HAL, est destinée au dépôt et à la diffusion de documents scientifiques de niveau recherche, publiés ou non, émanant des établissements d'enseignement et de recherche français ou étrangers, des laboratoires publics ou privés. 


\title{
Interval Estimation for LPV Systems Applying High Order Sliding Mode Techniques
}

\author{
Denis Efimov $^{\text {a }}$, Leonid Fridman ${ }^{\text {b }}$, Tarek Raïssi ${ }^{\mathrm{c}}$, Ali Zolghadri ${ }^{\mathrm{d}}$, Ramatou Seydou ${ }^{\mathrm{d}}$ \\ ${ }^{a}$ Non-A project at INRIA - LNE, Parc Scientifique de la Haute Borne, 40 avenue Halley, Bt.A Park Plaza, 59650 Villeneuve \\ d'Ascq, France \\ ${ }^{\mathrm{b}}$ Dept. de Ingeniera de Control y Robtica, Facultad de Ingeniera UNAM, Edificio "Bernardo Quintana", Ciudad \\ Universitaria D.F., México \\ ${ }^{\mathrm{c}}$ Conservatoire National des Arts et Mtiers (CNAM), Cedric-laetitia 292, Rue St-Martin, 75141 Paris, France \\ ${ }^{\mathrm{d}}$ University of Bordeaux, IMS-lab, Automatic control group, 351 cours de la libration, 33405 Talence, France
}

\begin{abstract}
In this paper, the problem of design of interval observers for Linear-Parameter-Varying (LPV) systems, containing non detectable or non strongly observable parts, is addressed. Firstly, a High Order Sliding Mode (HOSM) method is applied to the strongly observable subsystem, obtained by an appropriate change of coordinates, to estimate the state and its derivative. Secondly, this information is used to decrease the level of uncertainty in the rest of the system, which leads to improvement of accuracy of the set-membership estimates generated by an interval observer. Moreover, it is shown that HOSM techniques allows us to relax the applicability conditions of standard interval observer design methods. The efficiency of the proposed approach is demonstrated through simulation examples.
\end{abstract}

Key words: Interval observers, Sliding-mode, Linear Parameter-Varying System

\section{Introduction}

The problem of state estimation for nonlinear systems is very challenging and has been extensively studied in the literature, see for instance $[20,10,5]$. Although a complete palette of solutions exists for linear systems, in the nonlinear case mainly particular approaches are available. Many solutions are based on the system representation in a canonical form (frequently, partially linear). For nonlinear systems, it has been shown that an LPV equivalent representation can be an appealing alternative to deal with the original nonlinear system $[18,27,30]$. The basic idea is to replace the nonlinear complexity of the original system by an enlarged parametric variation in the LPV representation, which may simplify the observer design. There are several approaches to design observers for LPV systems $[4,13,14,21]$. The work pre-

Email addresses: denis.efimov@inria.fr (Denis Efimov), lfridman@servidor.unam.mx (Leonid Fridman), tarek.raissi@cnam.fr (Tarek Raïssi),

Ali.Zolghadri@ims-bordeaux.fr (Ali Zolghadri), Seydou.Ramatou@ims-bordeaux.fr (Ramatou Seydou). sented in this paper falls within the scope of interval observers [4,21]. That approach has been recently extended in [26] to nonlinear systems using LPV representation with known minorant and majorant matrices, and in [25] for observable nonlinear systems relaxing requirement on cooperativity (monotonicity) of the original system dynamics. Basically, the interval observers propagate the parameter uncertainty in the width of the estimated interval for the state values. So, the length of interval determines the estimation accuracy of the approach. This is why the uncertainty decreasing is very important for improvement of the interval (set-membership) estimation performance. This key feature is the subject studied in this paper.

On the other hand, the HOSM techniques have become very popular for design of observers for linear and nonlinear systems $[1,3,22,7,24,28]$. The sliding modes ensure a finite time of the estimation error convergence to zero and complete insensitivity to a matched uncertainty $[6,2,23]$. Mainly these features can be achieved under assumption that the system is strongly observable or strongly detectable [3]. The objective of this work is to 
combine both approaches (the interval observers and the HOSM techniques) in order to improve accuracy of estimation achieved by interval observers. The idea is that under a linear transformation of coordinates, an LPV system always has a strongly observable subsystem. Applying HOSM differentiation approach it is possible to estimate the state and the state derivative for this subsystem, which can be further used for improved evaluation of the input and the parameter uncertainty in the rest part of the system. This combination leads to a significant decrease of the interval estimation conservatism. Moreover, a relaxation of some applicability constraints usually met in interval estimation can be obtained.

The paper is organized as follows. The next section is devoted to some preliminaries. The main result is described in Section 3. Finally, Section 4 provides some simulation results.

\section{Preliminaries}

Euclidean norm for a vector $x \in \mathbb{R}^{n}$ will be denoted as $|x|$, and for a measurable and locally essentially bounded input $u: \mathbb{R}_{+} \rightarrow \mathbb{R}\left(\mathbb{R}_{+}=\{\tau \in \mathbb{R}: \tau \geq 0\}\right)$ the symbol $\|u\|_{\left[t_{0}, t_{1}\right]}$ denotes its $L_{\infty}$ norm:

$$
\|u\|_{\left[t_{0}, t_{1}\right]}=e s s \sup \left\{|u(t)|, t \in\left[t_{0}, t_{1}\right]\right\}
$$

if $t_{1}=+\infty$ then we will simply write $\|u\|$. Denote by $\mathcal{L}_{\infty}$ the set of all inputs $u$ satisfying $\|u\|<\infty$, and the sequence of integers $1, \ldots, k$ by $\overline{1, k}$.

In this work we consider the following LPV representation of a nonlinear system:

$$
\begin{gathered}
\dot{x}=A(\theta(t)) x+B(\theta(t)) u(t), \\
y=C x, \psi(t)=y+v(t),
\end{gathered}
$$

where $x \in \mathbb{R}^{n}, u \in \mathbb{R}^{m}, y \in \mathbb{R}^{p}, v \in \mathbb{R}^{p}$ are the state, the input, the output and the measurement noise of the system (1), $\psi(t)$ is the signal available for on-line measurements; $\theta \in \Theta \subset \mathbb{R}^{q}$ is the scheduling parameter vector, the set $\Theta$ is known; the matrix functions $A: \Theta \rightarrow \mathbb{R}^{n \times n}$ and $B: \Theta \rightarrow \mathbb{R}^{n \times m}$ are given. The instant values of $u(t) \in \mathcal{L}_{\infty}, v(t) \in \mathcal{L}_{\infty}$ and $\theta(t) \in \mathcal{L}_{\infty}$ are not known. Almost all existent approaches assume that the vector $\theta$ is accessible for measurements, in the following this assumption is relaxed, and only the domain $\Theta$ is given.

Assumption $1\|x\| \leq X,\|u\| \leq U$ and $\|v\| \leq V$, the bounds $X>0, U>0$ and $V>0$ are given.

Boundedness of the state $x$ and the inputs $u, v$ is a standard assumption in the estimation theory. Under Assumption 1 the signal $\psi(t)$ is also bounded.

\subsection{HOSM differentiation}

Taking the $s$-th time differentiable output $y(t)$ of the system (1), its derivatives can be estimated by the HOSM differentiator $[16,17]$ :

$$
\begin{gathered}
\dot{q}_{0}=\nu_{0}, \nu_{0}=-\lambda_{0}\left|q_{0}-\psi(t)\right|^{s / s+1} \operatorname{sign}\left[q_{0}-\psi(t)\right]+q_{1} \\
\dot{q}_{i}=\nu_{i}, i=\overline{1, s-1} \\
\nu_{i}=-\lambda_{i}\left|q_{i}-\nu_{i-1}\right|^{s-i / s-i+1} \operatorname{sign}\left[q_{i}-\nu_{i-1}\right]+q_{i+1} \\
\dot{q}_{s}=-\lambda_{s} \operatorname{sign}\left[q_{s}-\nu_{s-1}\right]
\end{gathered}
$$

where $\lambda_{k}, k=\overline{0, s}$ are positive parameters to be tuned (the procedure for their choice as a function of an upper estimate of $\psi^{(s)}(t)$ is discussed in [17,12], an on-line adaptation algorithm for their adjustment can be found in $[7])$.

Theorem 1 [17] Let $y: \mathbb{R}_{+} \rightarrow \mathbb{R}$ be s-th times continuously differentiable and $v(t) \in \mathcal{L}_{\infty}$ in (1), then there exist $0 \leq T<+\infty$ and some constants $\mu_{k}>0, k=\overline{0, s}$ (dependent on $\lambda_{k}, k=\overline{0, s}$ only) such that in (2) for all $t \geq T$ :

$$
\left|q_{k}(t)-y^{(k)}(t)\right| \leq \mu_{k}|| v \|^{\frac{s-k+1}{s+1}}, k=\overline{0, s} .
$$

In particular, this result means that if $v(t) \equiv 0$ for all $t \geq 0$, then the differentiator (2) ensures the exact estimation of derivatives in a finite time. Application of HOSM differentiators for unknown input estimation and compensation in linear systems has been studied in [3], an extension to nonlinear systems is presented in [9].

\subsection{Interval estimation}

For two vectors $x_{1}, x_{2} \in \mathbb{R}^{n}$ or matrices $A_{1}, A_{2} \in \mathbb{R}^{n \times n}$, the relations $x_{1} \leq x_{2}$ and $A_{1} \leq A_{2}$ are understood elementwise. Given a matrix $A \in \mathbb{R}^{m \times n}$ or a vector $x \in$ $\mathbb{R}^{n}$, define $A^{+}=\max \{0, A\}, A^{-}=A^{+}-A$ or $x^{+}=$ $\max \{0, x\}, x^{-}=x^{+}-x$ respectively.

Lemma 2 Let $x \in \mathbb{R}^{n}$ be a vector variable, $\underline{x} \leq x \leq \bar{x}$ for some $\underline{x}, \bar{x} \in \mathbb{R}^{n}$.

1) If $A \in \mathbb{R}^{m \times n}$ is a constant matrix, then

$$
A^{+} \underline{x}-A^{-} \bar{x} \leq A x \leq A^{+} \bar{x}-A^{-} \underline{x} .
$$

2) If $A \in \mathbb{R}^{m \times n}$ is a matrix variable, $\underline{A} \leq A \leq \bar{A}$ for some $\underline{A}, \bar{A} \in \mathbb{R}^{m \times n}$, then

$$
\begin{gathered}
\underline{A}^{+} \underline{x}^{+}-\bar{A}^{+} \underline{x}^{-}-\underline{A}^{-} \bar{x}^{+}+\bar{A}^{-} \bar{x}^{-} \leq A x \leq \\
\bar{A}^{+} \bar{x}^{+}-\underline{A}^{+} \bar{x}^{-}-\bar{A} \underline{x}^{+}+\underline{A}^{-} \underline{x}^{-} .
\end{gathered}
$$


Proof. To prove the first part note that $A x=\left(A^{+}-\right.$ $\left.A^{-}\right) x$, which for $x \leq x \leq \bar{x}$ gives the required inequalities. The proof of the second part is based on (3), where $A^{+}$and $A^{-}$are the functions with $\underline{A} \leq A \leq \bar{A}$. The relations (3) can be rewritten as follows:

$$
\begin{gathered}
A^{+}\left(\underline{x}^{+}-\underline{x}^{-}\right)-A^{-}\left(\bar{x}^{+}-\bar{x}^{-}\right) \leq A x \\
\leq A^{+}\left(\bar{x}^{+}-\bar{x}^{-}\right)-A^{-}\left(\underline{x}^{+}-\underline{x}^{-}\right),
\end{gathered}
$$

which for $\underline{A} \leq A \leq \bar{A}$ gives the desired inequalities.

A matrix $A \in \mathbb{R}^{n \times n}$ is called Hurwitz if all its eigenvalues have a negative real part, it is called Metzler if all its elements outside the main diagonal are nonnegative. Any solution of the linear system

$$
\dot{x}=A x+\omega(t), \omega: \mathbb{R}_{+} \rightarrow \mathbb{R}_{+}^{n},
$$

with $x \in \mathbb{R}^{n}$ and a Metzler matrix $A$, is elementwise nonnegative for all $t \geq 0$ provided that $x(0) \geq 0$ [29]. Such dynamical systems are called cooperative (monotone) [29].

\section{Main result}

For brevity of presentation the case $p=1$ is considered only (the case of vector measurements can be treated similarly). We will need the following assumptions.

Assumption 2 For all $\theta \in \Theta$, there is an invertible matrix $S(\theta) \in \mathbb{R}^{n \times n}$ such that the system (1) can be represented as follows:

$$
\begin{gathered}
x=S(\theta)\left[\begin{array}{c}
z_{1} \\
z_{2}
\end{array}\right], y=c^{T} z_{1}, \\
\operatorname{dim}\left\{z_{1}\right\}=n_{1}, \operatorname{dim}\left\{z_{2}\right\}=n_{2}, n_{1}+n_{2}=n, \\
\dot{z}_{1}=A_{0} z_{1}+b_{0}\left[a_{11}(\theta)^{T} z_{1}+a_{12}(\theta)^{T} z_{2}+b_{1}(\theta)^{T} u\right], \\
\dot{z}_{2}=A_{21}(\theta) z_{1}+A_{22}(\theta) z_{2}+B_{2}(\theta) u,
\end{gathered}
$$

where

$$
\begin{aligned}
& c=\left[\begin{array}{lll}
1 & 0 \ldots .0
\end{array}\right]^{T}, b_{0}=\left[\begin{array}{lll}
0 & \ldots & 1
\end{array}\right]^{T}, \\
& A_{0}=\left[\begin{array}{cccc}
0 & 1 & \ldots & 0 \\
\vdots & \ddots & \vdots \\
0 & 0 \ldots 0 & 1 \\
0 & 0 & \ldots & 0
\end{array}\right]
\end{aligned}
$$

is a canonical representation, the vector functions $a_{11}(\theta)$, $a_{12}(\theta), b_{1}(\theta)$ and the matrix functions $A_{21}(\theta), A_{22}(\theta)$, $B_{2}(\theta)$ have corresponding dimensions.
Assumption 2 states that there exists a transformation coordinates, which represents the system (1) as a pair of interconnected subsystems (5) and (6). The subsystem (5) is strongly observable since it has the canonical representation $c, A_{0}, b_{0}$ (the conditions of existence of such a transformation for linear time-invariant systems are analyzed in [3]). However, the system is not necessarily detectable (the dynamics of (1) could be non-minimum phase as in [28]) since there is no requirement on stability of the matrix function $A_{22}(\theta)$. This relaxation may be important for application of an interval observer design method for estimation in uncertain non-minimum phase systems. It is worth to stress that for $n_{1}=1$ this assumption is always true (at least the output coordinate can be chosen in the vector $z_{1}$, i.e. the linear systems always have a strongly observable subsystem).

Remark 3 Instability of the matrix function $A_{22}(\theta)$ does not contradict Assumption 1 (with the boundedness of the state $x$, and the inputs $u, v)$. Indeed, as we will show below (see Remark 7) this assumption can be introduced on a finite time interval only, then the system can be unstable (as in the third example). In addition, the matrix $A_{22}(\theta)$ is uncertain and possibly time-varying, then due to the incertitude it may be neither stable nor unstable (as in the first example, where for the chosen initial conditions the system has bounded trajectories). Finally, the system may be non-minimum phase or unstable, but the control input u may stabilize it guaranteeing Assumption 1, while for the observer synthesis the expression of $u$ is not available and only the signal $u(t)$ is measured (the control law $u$ includes some variables which are not accessible for a locally designed observer in the distributed system, for example). Thus in all these cases the matrix $A_{22}(\theta)$ can be unstable, but the system trajectories are bounded (at least locally in time) and Assumption 1 is satisfied.

Assumption 3 Let there exist a vector function $f(\theta) \in$ $\mathbb{R}^{n_{2}}$ such that

$$
\begin{gathered}
{\left[A_{22}(\theta) z_{2}+B_{2}(\theta) u\right]-f(\theta)\left[a_{12}(\theta)^{T} z_{2}+b_{1}(\theta)^{T} u\right]} \\
=\Delta_{1} z_{2}+\Delta_{2}(\theta) u
\end{gathered}
$$

for some Hurwitz matrix $\Delta_{1} \in \mathbb{R}^{n_{2} \times n_{2}}$ and $\Delta_{2}: \Theta \rightarrow$ $\mathbb{R}^{n_{2} \times m}$.

This assumption states that the matrix $\Delta_{1}=A_{22}(\theta)-$ $f(\theta) a_{12}(\theta)^{\mathrm{T}}$ is Hurwitz, i.e. the matrix $A_{22}(\theta)$ can be stabilized by an output feedback or the pair of matrices $\left(A_{22}(\theta), a_{12}(\theta)^{\mathrm{T}}\right)$ is observable for all $\theta \in \Theta$, and independent in $\theta$ (for an LMI verification of stability and observability of interval matrices see the recent work [15] and references therein).

Assumption 4 There exists a matrix $P \in \mathbb{R}^{n_{2} \times n_{2}}$ such that the matrix $D=P^{-1} \Delta_{1} P$ is Hurwitz and Metzler $(H \& M)$. 
Under mild conditions of the main result in [25], in the case of Assumption 3 there is a matrix $P \in \mathbb{R}^{n_{2} \times n_{2}}$ such that $D$ is $\mathrm{H} \& \mathrm{M}$, as it is stated in Assumption 4 (the proposed in [25] approach to calculate the matrix $P$ is based on solution of a Sylvester equation). In the paper [19] it is shown that always there exists a time varying similarity transformation $P(t)$ such that $D=$ $P(t)^{-1} \Delta_{1} P(t)$ for an H\&M matrix $D$.

Remark 4 The introduced assumptions are less restrictive than usually stated to design a HOSM or an interval observer. Indeed, an application of the techniques from [2,24,28] for (5), (6) is blocked due to the dependence on $\theta$ of all matrices in the system equations. The solutions from [4,21] are hard to apply for a non-detectable and non-cooperative system. However, a combination of both approaches allows us to design an interval observer for the LPV system (5), (6) with a non-minimum phase internal dynamics.

Under these assumptions it is proposed to use the differentiator (2) to estimate the state $z_{1}$ and its derivative $\dot{z}_{1}$, then from (5) we get an improved estimate on the signal $a_{12}(\theta)^{\mathrm{T}} z_{2}+b_{1}(\theta)^{\mathrm{T}} u$, which can be applied for design of an interval observer for the system (6) in the new coordinates $r=P^{-1} z_{2}$. Let us consider these steps consequently.

Under Assumption 2 the output $y$ of the system (5) has $n_{1}$ derivatives. Therefore according to Theorem 1 and Assumption 1, there exist parameters $\lambda_{k}, k=\overline{0, n_{1}}$ in (2) with $s=n_{1}$ and $T>0$ such that for all $t \geq T$ :

$$
\left|q_{k}(t)-y^{(k)}(t)\right| \leq \mu_{k} V^{\frac{n_{1}-k+1}{n_{1}+1}}, k=\overline{0, n_{1}}
$$

for some constant $\mu_{k}, k=\overline{0, n_{1}}$. Thus $z_{1}(t)=\hat{z}_{1}(t)+$ $e_{1}(t)$ and $\dot{z}_{1, n_{1}}(t)=q_{n_{1}}(t)+e_{2}(t)$ for all $t \geq T$, where $\hat{z}_{1, i}(t)=q_{i-1}(t)$ and $\left|e_{1, i}(t)\right| \leq \mu_{i-1} V^{\frac{n_{1}-i+2}{n_{1}+1}}$ for $i=$ $\overline{1, n_{1}},\left|e_{2}(t)\right| \leq \mu_{n_{1}} V^{\frac{1}{n_{1}+1}}$. The variables $\hat{z}_{1}$ and $q_{n_{1}}$ are generated by the HOSM differentiator (2), thus they are available for a designer. The errors $e_{1}$ and $e_{2}$ are upper bounded by some functions of $V$. Substitution of these variables into the last equation of (5) gives $q_{n_{1}}+e_{2}=$ $a_{11}(\theta)^{\mathrm{T}}\left[\hat{z}_{1}+e_{1}\right]+a_{12}(\theta)^{\mathrm{T}} z_{2}+b_{1}(\theta)^{\mathrm{T}} u$, or equivalently

$$
a_{12}(\theta)^{\mathrm{T}} z_{2}+b_{1}(\theta)^{\mathrm{T}} u=q_{n_{1}}+e_{2}-a_{11}(\theta)^{\mathrm{T}}\left[\hat{z}_{1}+e_{1}\right]
$$

Substituting this equality in the differential equation (6) we obtain

$$
\begin{aligned}
\dot{z}_{2}= & \Delta_{1} z_{2}+\left[A_{21}(\theta)-f(\theta) a_{11}(\theta)^{\mathrm{T}}\right]\left(\hat{z}_{1}+e_{1}\right)+ \\
& f(\theta)\left(q_{n_{1}}+e_{2}\right)+\Delta_{2}(\theta) u
\end{aligned}
$$

which is a stable system according to Assumption 3.
Applying the transformation of coordinates $r=P^{-1} z_{2}$, the system (7) can be rewritten as follows

$$
\begin{gathered}
\dot{r}=D r+G_{1}(\theta)\left(\hat{z}_{1}+e_{1}\right)+G_{2}(\theta)\left(q_{n_{1}}\right. \\
\left.+e_{2}\right)+G_{3}(\theta) u,
\end{gathered}
$$

where $G_{1}(\theta)=P^{-1}\left[A_{21}(\theta)-f(\theta) a_{11}(\theta)^{\mathrm{T}}\right], G_{2}(\theta)=$ $P^{-1} f(\theta)$ and $G_{3}(\theta)=P^{-1} \Delta_{2}(\theta)$. The dynamics of $(8)$ is cooperative and stable, and all uncertain functions or variables in the right hand side of (8) belong to an interval for $\theta \in \Theta$ :

$$
\begin{gathered}
\underline{G_{j}} \leq G_{j}(\theta) \leq \overline{G_{j}}, j=\overline{1,3} ;|u(t)| \leq U ; \\
\left|e_{1, i}(t)\right| \leq \bar{e}_{1, i}=\mu_{i-1} V^{\frac{n_{1}-i+2}{n_{1}+1}}, i=\overline{1, n_{1}} ; \\
\left|e_{2}(t)\right| \leq \bar{e}_{2}=\mu_{n_{1}} V^{\frac{1}{n_{1}+1}}
\end{gathered}
$$

for all $t \geq T$, where the matrices $G_{j}, \overline{G_{j}}, j=\overline{1,3}$ are known. Therefore the following interval observer can be synthesized for $(8)$ :

$$
\begin{aligned}
& \dot{\bar{r}}=D \bar{r}+\left({\overline{G_{1}}}^{+}-{\overline{G_{1}}}^{-}\right) \hat{z}_{1}^{+}+\left(\underline{G_{1}}-{\underline{G_{1}}}^{+}\right) \hat{z}_{1}^{-}+ \\
& \left({\overline{G_{1}}}^{+}+{\underline{G_{1}}}^{-}\right) \bar{e}_{1}+\left({\overline{G_{2}}}^{+}-{\overline{G_{2}}}^{-}\right) q_{n_{1}}^{+}+ \\
& \left(\underline{G 2}^{-}-{\underline{G_{2}}}^{+}\right) q_{n_{1}}^{-}+\left({\overline{G_{2}}}^{+}+{\underline{G_{2}}}^{-}\right) \bar{e}_{2}+\left({\overline{G_{3}}}^{+}+{\underline{G_{3}}}^{-}\right) U \text {, } \\
& \underline{\dot{r}}=D \underline{r}+\left({\underline{G_{1}}}^{+}-{\underline{G_{1}}}^{-}\right) \hat{z}_{1}^{+}+\left({\overline{G_{1}}}^{-}-{\overline{G_{1}}}^{+}\right) \hat{z}_{1}^{-}- \\
& \left({\overline{G_{1}}}^{+}+{\underline{G_{1}}}^{-}\right) \bar{e}_{1}+\left({\underline{G_{2}}}^{+}-{\underline{G_{2}}}^{-}\right) q_{n_{1}}^{+}+ \\
& \left({\overline{G_{2}}}^{-}-{\overline{G_{2}}}^{+}\right) q_{n_{1}}^{-}-\left({\overline{G_{2}}}^{+}+{\underline{G_{2}}}^{-}\right) \bar{e}_{2}-\left({\overline{G_{3}}}^{+}+\underline{G_{3}}{ }^{-}\right) U \text {, }
\end{aligned}
$$

the properties (3), (4) have been used to calculate (9), (10). Introducing the interval estimation errors $\bar{\epsilon}=\bar{r}-r$, $\underline{\epsilon}=r-\underline{r}$, we obtain

$$
\dot{\bar{\epsilon}}=D \bar{\epsilon}+\bar{\varepsilon}, \underline{\dot{\epsilon}}=D \underline{\epsilon}+\underline{\varepsilon},
$$

where $\bar{\varepsilon}=\left({\overline{G_{1}}}^{+}-{\overline{G_{1}}}^{-}\right) \hat{z}_{1}^{+}+\left({\underline{G_{1}}}^{-}-{\underline{G_{1}}}^{+}\right) \hat{z}_{1}^{-}+\left({\overline{G_{1}}}^{+}+\right.$ \left.${\underline{G_{1}}}^{-}\right) \bar{e}_{1}+\left({\overline{G_{2}}}^{+}-{\overline{G_{2}}}^{-}\right) q_{n_{1}}^{+}+\left({\underline{G_{2}}}^{-}-{\underline{G_{2}}}^{+}\right) q_{n_{1}}^{-}+\left({\overline{G_{2}}}^{+}+\right.$ \left.${G_{2}}^{-}\right) \bar{e}_{2}+\left({\overline{G_{3}}}^{+}+G_{3}{ }^{-}\right) U-G_{1}(\theta)\left(\hat{z}_{1}+e_{1}\right)-G_{2}\left(q_{n_{1}}+e_{2}\right)-$ $\overline{G_{3}}(\theta) u, \underline{\varepsilon}=G_{1}\left(\overline{\theta)}\left(\hat{z}_{1}+e_{1}\right)+G_{2}\left(q_{n_{1}}+e_{2}\right)+G_{3}(\theta) u-\right.$ $\left({\underline{G_{1}}}^{+}-{\underline{G_{1}}}^{-}\right) \hat{z}_{1}^{+}-\left({\overline{G_{1}}}^{-}-{\overline{G_{1}}}^{+}\right) \hat{z}_{1}^{-}+\left({\overline{G_{1}}}^{+}+{\underline{G_{1}}}^{-}\right) \bar{e}_{1}+$ $\left({\underline{G_{2}}}^{+}-{\overline{G_{2}}}^{-}\right) q_{n_{1}}^{+}+\left({\overline{G_{2}}}^{-}-{\overline{G_{2}}}^{+}\right) q_{n_{1}}^{-}-\left({\overline{G_{2}}}^{+}+{\underline{G_{2}}}^{-}\right) \bar{e}_{2}+$ $\left(\overline{G_{3}}+{\overline{G_{3}}}^{-}\right) U$. It is an arithmetic exercise to verify that under assumptions 1 and 2 (and the result of Theorem 1) the residual terms $\bar{\varepsilon}$ and $\underline{\varepsilon}$ are elementwise positive and bounded. Then using the results of monotone system theory [29] we prove that for all $t \geq T$

$$
\underline{r}(t) \leq r(t) \leq \bar{r}(t)
$$

and the estimates $\underline{r}(t), \bar{r}(t)$ are bounded, provided that

$$
\underline{r}(T) \leq r(T) \leq \bar{r}(T) \text {. }
$$


The former relation for the initial conditions can be easily satisfied since $\|x\| \leq X$ under Assumption 1. Using the property (3) we get for all $t \geq T$ :

$$
\begin{gathered}
\underline{z}_{2}(t) \leq z_{2}(t)=\operatorname{Pr}(t) \leq \bar{z}_{2}(t), \\
\underline{z}_{2}(t)=P^{+} \underline{r}(t)-P^{-} \bar{r}(t), \bar{z}_{2}(t)=P^{+} \bar{r}(t)-P^{-} \underline{r}(t) ; \\
\underline{z}_{1}(t) \leq z_{1}(t) \leq \bar{z}_{1}(t) \\
\underline{z}_{1}(t)=\hat{z}_{1}(t)-\bar{e}_{1}, \bar{z}_{1}(t)=\hat{z}_{1}(t)+\bar{e}_{1} .
\end{gathered}
$$

Defining $\underline{z}=\left[\underline{z}_{1}^{\mathrm{T}} \underline{z}_{2}^{\mathrm{T}}\right]^{\mathrm{T}}, \bar{z}=\left[\bar{z}_{1}^{\mathrm{T}} \bar{z}_{2}^{\mathrm{T}}\right]^{\mathrm{T}}$ and using (4) we can formulate the interval estimates for the state $x$ :

$$
\begin{gathered}
\underline{S}^{+} \underline{z}^{+}-\bar{S}^{+} \underline{z}^{-}-\underline{S}^{-} \bar{z}^{+}+\bar{S}^{-} \bar{z}^{-} \leq x=S(\theta) z \leq \\
\bar{S}^{+} \bar{z}^{+}-\underline{S}^{+} \bar{z}^{-}-\bar{S}^{-} \underline{z}^{+}+\underline{S}^{-} \underline{z}^{-},
\end{gathered}
$$

which is satisfied for all $t \geq T$. Thus we have proven the following theorem.

Theorem 5 Let assumptions 1, 2, 3, 4 hold for the system (1). Then there exist the set of parameters $\lambda_{k}, k=$ $\overline{0, n_{1}}$ in (2) and a constant $T>0$ such that for all $t \geq T$ the interval estimate (12) is true, provided that the condition (11) is satisfied for (9), (10).

Remark 6 The assumptions 3 and 4 can be skipped if we would assume the existence of a vector function $f(\theta) \in$ $\mathbb{R}^{n_{2}}$ such that

$$
\begin{gathered}
{\left[A_{22}(\theta) z_{2}+B_{2}(\theta) u\right]-f(\theta)\left[a_{12}(\theta)^{T} z_{2}+b_{1}(\theta)^{T} u\right]} \\
=\Delta_{1}(\theta) z_{2}+\Delta_{2}(\theta) u
\end{gathered}
$$

for some Hurwitz and Metzler matrix function $\Delta_{1}: \Theta \rightarrow$ $\mathbb{R}^{n_{2} \times n_{2}}$ and some $\Delta_{2}: \Theta \rightarrow \mathbb{R}^{n_{2} \times m}$. Next, the result of Theorem 5 can be obtained using the same technique and an interval observer from the paper [26].

Remark 7 Since the interval estimates are obtained in a finite time, then Assumption 1 can be relaxed introducing the requirement on boundedness of the state $x$ during a finite time interval only for $t \in\left[0, T_{x}\right)$ with $T_{x} \leq T$, where $T$ is defined in Theorem 5.

Implicitly the conditions of Theorem 5 mean that the interval observer (9), (10) has to be activated for $t \geq$ $T$ only. The time $T$ can be detected on-line using the property:

$$
\sup _{t \geq T}\left|q_{0}(t)-\psi(t)\right| \leq \vartheta,
$$

where $\vartheta>0$ is a constant dependent on discretization step used for computation of $(2)(\vartheta=0$ under assumption that the differential equation (2) is solved without a computational error) [17].

\section{Examples}

To illustrate improvement achieved in interval estimation by application of HOSM techniques consider three examples. Two examples deal with non-minimum phase systems, the conventional techniques for the interval observer design $[4,21,25]$ cannot be applied in this case. And one minimum phase example is presented in order to compare the estimation accuracy for different interval observers.

\subsection{Non-minimum phase second order system}

In order to explain the peculiarities of the proposed solution, let us start with analysis of a second order academic example:

$$
\begin{aligned}
\dot{x}_{1} & =-a_{11}(\theta) x_{1}+a_{12}(\theta) x_{2}+b_{1}(\theta) u \\
\dot{x}_{2} & =a_{21}(\theta) x_{1}+a_{22}(\theta) x_{2}+b_{2}(\theta) u \\
y & =x_{1}
\end{aligned}
$$

where $x_{1} \in \mathbb{R}, x_{2} \in \mathbb{R}$ are the state variables, and for all $\theta \in \Theta$

$$
0.5 \leq a_{11}(\theta) \leq 1.5,1 \leq a_{12}(\theta) \leq 3,
$$

$-1 \leq a_{21}(\theta) \leq 1,-0.5 \leq a_{22}(\theta)=0.5 a_{12}(\theta)-1 \leq 0.5$, $0.75 \leq b_{1}(\theta) \leq 1,0.5 \leq b_{2}(\theta) \leq 1, U=0.25, V=0.03$.

As we can see, the system (13) is already in the form (5), (6) with $x_{1}=z_{1}$ and $x_{2}=z_{2}$ (the matrix $S(\theta)$ equals to the identity, and Assumption 2 is satisfied). For simulation we use

$$
\begin{gathered}
a_{11}(\theta)=1+0.5 \sin \left(3 x_{2} t\right), a_{12}(\theta)=2+\sin \left(2 x_{1} t\right), \\
a_{21}(\theta)=\sin (t), b_{1}(\theta)=0.5, b_{2}(\theta)=0.875+0.125 \cos (0.5 t), \\
u(t)=U \sin (2 t), v(t)=V \sin (10 t), \\
\theta=\left[x_{1} x_{2} t\right]^{\mathrm{T}} .
\end{gathered}
$$

For the system (13) with the chosen parameters and the given input $u$ the state is bounded (Assumption 1 holds). The initial condition uncertainty is $-4 \leq x_{2}(0) \leq 4$. It is easy to verify that for $f=0.5$ we have

$\left[a_{22}(\theta) z_{2}+b_{2}(\theta) u\right]-f\left[a_{12}(\theta) z_{2}+b_{1}(\theta) u\right]=\Delta_{1} z_{2}+\Delta_{2}(\theta) u$

for $\Delta_{1}=-1$ and $0.25 \leq \Delta_{2}(\theta) \leq 0.625$ (Assumption 3 is satisfied). Since $\Delta_{1}<0$ Assumption 4 is true with the matrix $P$ equals the identity.

Therefore, according to Theorem 5 we may use the differentiator (2) to estimate $x_{1}$ and $\dot{x}_{1}$, which for $s=2$ can be reduced to the conventional super-twisting differentiator $[16]$ :

$\dot{q}_{0}=-\lambda_{0} \sqrt{\left|q_{0}-\psi(t)\right|} \operatorname{sign}\left[q_{0}-\psi(t)\right]+q_{1} ;$

$\dot{q}_{1}=-\lambda_{1} \operatorname{sign}\left[q_{0}-\psi(t)\right]$,

where in our example $\lambda_{0}=20, \lambda_{1}=50, \hat{z}_{1}=q_{0}$ and $\bar{e}_{1}=1.1 \mathrm{~V}, \bar{e}_{2}=1.1 \sqrt{V}$. In this case the finite time 


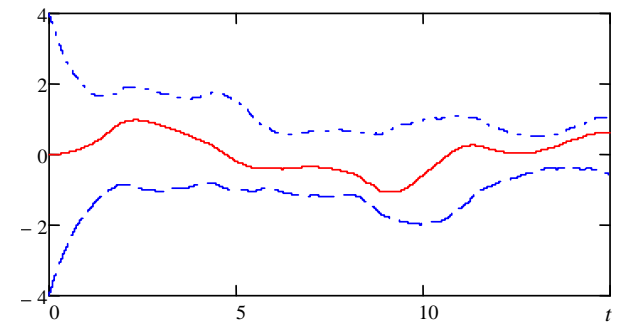

Fig. 1. The results of simulation for non-minimum phase example

$T=0.1$. Next, the interval observer (9), (10) generates the required set-membership estimates for the variable $x_{2}=r\left(-0.75 \leq G_{1}(\theta)=P^{-1}\left[a_{21}(\theta)+f a_{11}(\theta)\right] \leq 1.75\right.$, $G_{2}=0.5$ and $\left.\bar{G}_{3}(\theta)=\Delta_{2}(\theta)\right)$ :

$$
\begin{gathered}
\dot{\bar{r}}=-\bar{r}+1.75 \hat{z}_{1}^{+}-0.75 \hat{z}_{1}^{-}+1.75 \bar{e}_{1}+0.5 q_{1} \\
+0.5 \bar{e}_{2}+0.625 U \\
\dot{r}=-\underline{r}-0.75 \hat{z}_{1}^{+}+1.75 \hat{z}_{1}^{-}-1.75 \bar{e}_{1}+0.5 q_{1} \\
-0.5 \bar{e}_{2}-0.625 U .
\end{gathered}
$$

The results of this interval estimation are shown in Fig 1 , where the red solid line represents the variable $x_{2}$, while the dash and the dash-dot blue lines correspond to interval estimates $\underline{x}_{2}$ and $\bar{x}_{2}$ respectively. The interval estimates $\underline{x}_{2}, \bar{x}_{2}$ envelop the state trajectory of the plant $x_{2}$ and at some time instants the trajectory $x_{2}$ touches the bounds $\underline{x}_{2}, \bar{x}_{2}$ justifying that the obtained estimates are not conservative. The width of the estimated interval $\left[\underline{x}_{2}, \bar{x}_{2}\right]$ is proportional to the current system incertitude.

It is worth to note that for the best knowledge of the authors, other existent approaches cannot solve the problem of interval estimation for (13). In particular, application of a conventional interval observer design method $[21,26]$ is blocked by the non-minimum phase condition $\left(-0.5 \leq a_{22}(\theta) \leq 0.5\right)$.

\subsection{Minimum phase second order system}

Now consider a second order system for which we can apply a conventional approach for design of interval observers. In this example we would like to show the estimation accuracy improvement. Consider the system (13) with

$$
\begin{gathered}
a_{11}(\theta)=0.5, a_{12}(\theta)=0, \\
-0.5 \leq a_{21}(\theta) \leq 0.5, a_{22}(\theta)=-1, \\
b_{1}(\theta)=2,0.5 \leq b_{2}(\theta) \leq 1, U=1, V=0.1
\end{gathered}
$$

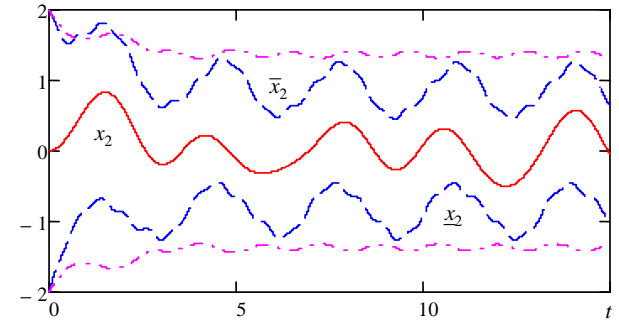

Fig. 2. The results of simulation for minimum phase example for $\theta \in \Theta$. Again Assumption 2 is satisfied. For simulation we use

$$
\begin{gathered}
a_{21}(\theta)=0.5 \sin (t), b_{2}(\theta)=0.75+0.25 \cos (0.5 t) \\
u(t)=U \sin (2 t), v(t)=V \sin (10 t)
\end{gathered}
$$

For the system (13) with the chosen parameters and the given input $u$ the state is bounded (Assumption 1 holds). We assume that $-2 \leq x_{2}(0) \leq 2$. For $f(\theta)=0.5 b_{2}(\theta)$ the equality $f(\theta) b_{1}(\theta)=b_{2}(\theta)$ holds (from the subsystem (5) we evaluate the value of $u$ ) and we have

$$
\left[a_{22}(\theta) z_{2}+b_{2}(\theta) u\right]-f(\theta)\left[a_{12}(\theta) z_{2}+b_{1}(\theta) u\right]=\Delta_{1} z_{2},
$$

where $\Delta_{1}=-1, \Delta_{2}(\theta)=0$ and Assumption 3 is satisfied. Since $\Delta_{1}<0$, as previously, Assumption 4 is true with the identity matrix $P$. For differentiation we again use (14) with the same values of parameters. In accordance with the result of Theorem 5 the interval observer takes form for $x_{2}=r\left(-0.25 \leq G_{1}(\theta)=\right.$ $P^{-1}\left[a_{21}(\theta)+f(\theta) a_{11}(\theta)\right] \leq 1,0.25 \leq G_{2}=f(\theta) \leq 0.5$ and $\left.G_{3}(\theta)=0\right)$ :

$$
\begin{aligned}
& \dot{\bar{r}}=-\bar{r}+\hat{z}_{1}^{+}-0.25 \hat{z}_{1}^{-}+\bar{e}_{1}+0.5 q_{1}^{+}+0.25 q_{1}^{-}+0.5 \bar{e}_{2}, \\
& \dot{\dot{r}}=-\underline{r}-0.25 \hat{z}_{1}^{+}+\hat{z}_{1}^{-}-\bar{e}_{1}+0.25 q_{1}^{+}+0.5 q_{1}^{-}-0.5 \bar{e}_{2} .
\end{aligned}
$$

Note that in this case for the $x_{2}$ subsystem an interval observer can be designed directly using [26] $\left(x_{2}=r\right.$ and $\left.x_{1}(t) \in[\psi(t)-V, \psi(t)+V]\right)$ :

$$
\begin{aligned}
& \dot{\bar{r}}=-\bar{r}+0.5|\psi|+0.5 V+U, \\
& \dot{\underline{r}}=-\underline{r}-0.5|\psi|-0.5 V-U .
\end{aligned}
$$

As we see the observer (16) depends directly on the worst case estimate $U$ for the input $u$, while the observer (15) is based on estimated value of the input $u$ calculated in the subsystem (5) using (14). Such a substitution leads to accuracy of estimation improvement as it is confirmed by the results of simulation for both observers presented in Fig. 2 (the solid line represents $x_{2}(t)$, the dash lines correspond to the interval estimates of the observer (15) and the dash-dot lines show the estimates calculated by $(16))$. 


\subsection{Pendulum system}

Consider the model of an inverted pendulum linearized around the upper unstable equilibrium:

$$
\begin{gathered}
\dot{x}_{1}=x_{2}, \dot{x}_{2}=-g m x_{3}-k_{1}(\theta) x_{2}+u(t) ; \psi=x_{1}+v ; \\
\dot{x}_{3}=x_{4}, \dot{x}_{4}=\ell^{-1}\left[g(M+m) x_{3}-k_{2} x_{4}+u(t)\right],
\end{gathered}
$$

where $x_{1} \in \mathbb{R}, x_{2} \in \mathbb{R}$ are deviations of the cart position $[\mathrm{m}]$ and velocity $[\mathrm{m} / \mathrm{sec}]$ respectively, $x_{3} \in[-\pi, \pi)$, $x_{4} \in \mathbb{R}$ are the angular position and velocity in $[\mathrm{rad} / \mathrm{sec}] ;$ $M$ and $m$ are known masses of the cart and the pendulum point respectively [kg], $\ell$ is a fixed known length of the pendulum link $[\mathrm{m}], g=9.8$ is the gravity acceleration $\left[\mathrm{m} / \mathrm{sec}^{2}\right], k_{2}>0$ and $\underline{k} \leq k_{1}(\theta) \leq \bar{k}$ are friction coefficients ( $k_{1}$ is unknown and time-varying); $u=u_{0}+d$ $\left[\mathrm{m} / \mathrm{sec}^{2}\right], u_{0} \in \mathbb{R}$ is an exciting input, $-\bar{d} \leq d \leq \bar{d}$ is an external bounded disturbance and $v \in \mathbb{R}$ is the measurement noise $[\mathrm{m}]$. The cart subsystem has one negative eigenvalue and zero eigenvalue, the pendulum subsystem has one positive and one negative eigenvalues (the system is unstable at the upper equilibrium). Only the cart position $x_{1}$ is available for measurements. The positive parameters $\bar{d}, \underline{k}$ and $\bar{k}$ are given.

For an exciting input $u_{0}$ the pendulum is unstable, but its solutions stay bounded during some small finite time interval. We are going to show that the proposed observer is able to generate the interval estimates in less than 0.1 [sec] that is acceptable for further stabilizing control application [11,8] (see also Remark 7). Another solution is to use a HOSM algorithm for bounded logarithmic derivatives (it is true for linear systems).

Obviously in this example the cart subsystem is strongly observable $\left(z_{1}=\left[\begin{array}{ll}x_{1} & x_{2}\end{array}\right]^{\mathrm{T}}, z_{2}=\left[\begin{array}{ll}x_{3} & x_{4}\end{array}\right]^{\mathrm{T}}\right)$ and using the HOSM differentiator of the third order $(s=2)$ we are able to estimate $x_{2}$ and $\dot{x}_{2}$ :

$$
x_{2}(t)=q_{1}(t)+e_{1}(t), \dot{x}_{2}(t)=q_{2}(t)+e_{2}(t) .
$$

Substitution of these estimates in the second equation gives:

$$
q_{2}(t)+e_{2}(t)+k_{1}(\theta)\left[q_{1}(t)+e_{1}(t)\right]-u_{0}=d-g m x_{3} .
$$

Using this relation the equations of the link subsystem can be rewritten as follows

$$
\begin{aligned}
\dot{x}_{3}= & x_{4}+f_{1}\left[d-g m x_{3}\right]- \\
& f_{1}\left[q_{2}+e_{2}+k_{1}(\theta)\left(q_{1}+e_{1}\right)-u_{0}\right], \\
\dot{x}_{4}= & \ell^{-1}\left[g\{M+m\} x_{3}-k_{2} x_{4}+u_{0}+d\right],
\end{aligned}
$$

where $f_{1}>0$ is chosen high enough to ensure that the Metzler matrix

$$
\left[\begin{array}{cc}
-f_{1} g m & 1 \\
\ell^{-1} g(M+m) & -\ell^{-1} k_{2}
\end{array}\right]
$$
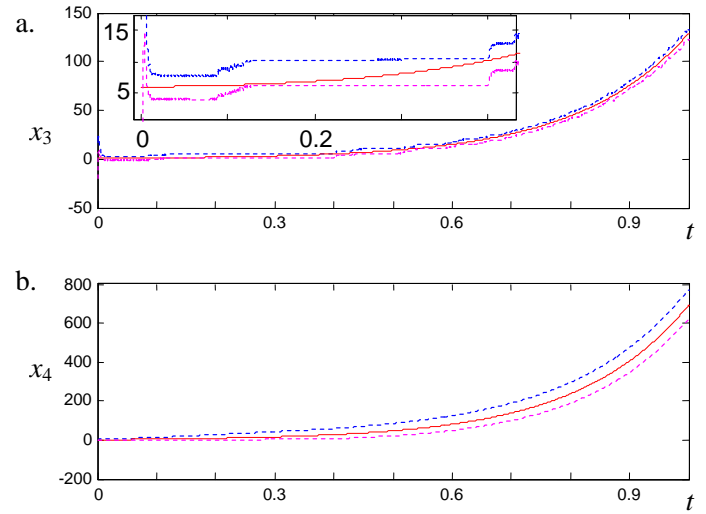

Fig. 3. The results of the angle position and velocity estimation

is Hurwitz (this matrix describes the dynamics of the link subsystem). In this case all conditions of the Theorem 5 are satisfied for $f(\theta)=\left[f_{1} 0\right]^{\mathrm{T}}$ and the interval observer takes form:

$$
\begin{aligned}
\underline{\dot{x}}_{3}= & \underline{x}_{4}+f_{1}\left[-\bar{d}-g m \underline{x}_{3}\right]- \\
& f_{1}\left[q_{2}+\bar{e}_{2}+\bar{k} q_{1}^{+}-\underline{k} q_{1}^{-}+\bar{k} \bar{e}_{1}-u_{0}\right], \\
\underline{x}_{4}= & \ell^{-1}\left[g\{M+m\} \underline{x}_{3}-k_{2} \underline{x}_{4}+u_{0}-\bar{d}\right], \\
\dot{\bar{x}}_{3}= & \bar{x}_{4}+f_{1}\left[\bar{d}-g m \bar{x}_{3}\right]- \\
& f_{1}\left[q_{2}-\bar{e}_{2}+\underline{k} q_{1}^{+}-\bar{k} q_{1}^{-}-\bar{k} \bar{e}_{1}-u_{0}\right], \\
\dot{\bar{x}}_{4}= & \ell^{-1}\left[g\{M+m\} \bar{x}_{3}-k_{2} \bar{x}_{4}+u_{0}+\bar{d}\right] .
\end{aligned}
$$

The results of this observer application for (the units are described above)

$$
\begin{gathered}
M=5, m=1, \ell=2, \bar{v}=0.02, \underline{k}=0.1, \bar{k}=0.4, \\
k_{1}=0.25+0.15 \sin \left(x_{2}^{2}\right), k_{2}=0.2, \\
u_{0}(t)=0.15 \sin (20 \pi t), v(t)=0.02 \sin (2 \pi t), \\
v(t)=0.01 \sin (5 \pi t), f_{1}=45, \bar{e}_{1}=0.1, \bar{e}_{2}=19
\end{gathered}
$$

are shown in Fig. 3, the results of interval estimation for the variable $x_{3}$ are given in Fig. 3 , a and for the variable $x_{4}$ in Fig. 3,b (solid lines represent the variables $x_{3}$ and $x_{4}$, the dash lines correspond to upper and lower estimates). As we can conclude from this figure, the pendulum is unstable, but the sliding-mode interval observer is able to evaluate the set of admissible values for both variables very quickly in a finite time (see the zoomed inclusion), this inforamtion can be used for control [8].

\section{Conclusion}

The objective of this technical note is to present an approach for improvement of estimation accuracy for interval observers designed for LPV systems. Applying the 
HOSM differentiation, the information from a strongly observable subsystem is used to decrease the level of uncertainty in the rest of the underlying system. That allow us to improve the estimation accuracy of an interval observer designed for LPV systems, and enlarge the class of LPV systems having an interval observer. The efficiency of the proposed technique has been demonstrated through non-minimum phase examples.

\section{References}

[1] J.-P. Barbot, D. Boutat, and T. Floquet. An observation algorithm for nonlinear systems with unknown inputs. Automatica, 45:1970-1974, 2009.

[2] G. Bartolini, L. Fridman, A. Pisano, and E. Usai, editors. Modern Sliding Mode Control Theory: New Perspectives and Applications, volume 375 of Lecture Notes in Control and Information Sciences. Springer, 2008.

[3] F. Bejarano and A. Pisano. Switched observers for switched linear systems with unknown inputs. IEEE Trans. Automatic Control, 56(3):681-686, 2011.

[4] O. Bernard and J.L. Gouz. Closed loop observers bundle for uncertain biotechnological models. J. Process Control, 14:765-774, 2004.

[5] G. Besanon, editor. Nonlinear Observers and Applications, volume 363 of Lecture Notes in Control and Information Sciences. Springer, 2007.

[6] C. Edwards and S.K. Spurgeon. Sliding mode control: theory and applications. Taylor \& Francis, 1998.

[7] D. Efimov and L. Fridman. Global sliding-mode observer with adjusted gains for locally lipschitz systems. Automatica, 47(3):565-570, 2011.

[8] D. Efimov, T. Raïssi, and A. Zolghadri. Stabilization of nonlinear uncertain systems based on interval observers. In Proc. IEEE CDC-ECC 2011, Orlando, FL, 2011.

[9] D. Efimov, A. Zolghadri, and T. Raïssi. Actuators fault detection and compensation under feedback control. Automatica, 47:1699-1705, 2011.

[10] T.I. Fossen and H. Nijmeijer, editors. New Directions in Nonlinear Observer Design. Springer, 1999.

[11] L. Fridman, A. Levant, and J. Davila. Observation of linear systems with unknown inputs via high-order sliding-mode. Int. J. Systems Science, 38(10):773-791, 2007.

[12] L. Fridman, J. Moreno, and R. Iriarte, editors. Sliding Modes after the first Decade of the 21st Century: State of the Art, volume 412 of Lecture Notes in Control and Information Sciences. Springer, 2011.

[13] L. Jaulin. Nonlinear bounded-error state estimation of continuous time systems. Automatica, 38(2):1079-1082, 2002.

[14] M. Kieffer and E. Walter. Guaranteed nonlinear state estimator for cooperative systems. Numerical Algorithms, 37:187-198, 2004.

[15] D.H. Lee, J.B. Park, and Y.H. Joo. A less conservative lmi condition for robust d-stability of polynomial matrix polytopes-a projection approach. IEEE Trans. Automatic Control, 56(4):868-873, 2010.

[16] A. Levant. Robust exact differentiation via sliding mode technique. Automatica, 34(3):379-384, 1998.

[17] A. Levant. High-order sliding modes: differentiation and output-feedback control. Int. J. Control, 76(9-10):924-941, 2003.
[18] A. Marcos and J. Balas. Development of linear-parametervarying models for aircraft. J. Guidance, Control, Dynamics, $27(2), 2004$.

[19] F. Mazenc and O. Bernard. Interval observers for linear timeinvariant systems with disturbances. Automatica, 47(1):140$147,2011$.

[20] T. Meurer, K. Graichen, and E.-D. Gilles, editors. Control and Observer Design for Nonlinear Finite and Infinite Dimensional Systems, volume 322 of Lecture Notes in Control and Information Sciences. Springer, 2005.

[21] M. Moisan, O. Bernard, and J.L. Gouz. Near optimal interval observers bundle for uncertain bio-reactors. Automatica, 45(1):291-295, 2009.

[22] J.A. Moreno and M. Osorio. Strict lyapunov functions for the super-twisting algorithm. IEEE Transactions on Automatic Control, 57(4):1035-1040, 2012.

[23] W. Perruquetti and J.P. Barbot, editors. Sliding mode control in engineering. Marcel Dekker, 2002.

[24] A. Pisano and E. Usai. Sliding mode control: a survey with applications in math. Mathematics and Computers in Simulation, 81:954-979, 2011.

[25] T. Raïssi, D. Efimov, and A. Zolghadri. Interval state estimation for a class of nonlinear systems. IEEE Trans. Automatic Control, 57(1):260-265, 2012.

[26] T. Raïssi, G. Videau, and A. Zolghadri. Interval observers design for consistency checks of nonlinear continuous-time systems. Automatica, 46(3):518-527, 2010.

[27] J. Shamma and J. Cloutier. Gain-scheduled missile autopilot design using linear parameter-varying transformations. $J$. Guidance, Control, Dynamics, 16(2):256-261, 1993.

[28] Yu.B. Shtessel, S. Baev, C. Edwards, and S. Spurgeon. Hosm observer for a class of non-minimum phase causal nonlinear mimo systems. IEEE Trans. Automatic Control, 55(2):543$548,2010$.

[29] H.L. Smith. Monotone Dynamical Systems: An Introduction to the Theory of Competitive and Cooperative Systems, volume 41 of Surveys and Monographs. AMS, Providence, 1995.

[30] W. Tan. Applications of Linear Parameter-Varying Control Theory. PhD thesis, Dept. of Mechanical Engineering, University of California at Berkeley, 1997. 